\title{
Inhalt des Jahrganges 42 / Sommaire du volume 42
}

Erwin H. Ackerknecht, Nachruf auf Karl Eduard Rothschuh 201

- Geburtstagsadresse für Jean Starobinski 211

- Somme remarks concerning bureaucracy and medicine 221

François Azouvi, Homo duplex $\quad 229$

Bronislaw Baczko, Le Cercle et la spirale 213

Luigi Belloni, Il «tumore» endorbitario del Feldmaresciallo Radetzky guarito «dalla
terapia omeopatica»

Isaac Benguigui, Charles-Gaspard de la Rive (1770-1834), médecin aliéniste et physicien 245

$\begin{array}{ll}\text { Urs Boschung, Zur Edition des Briefwechsels Haller-Bonnet } & 167\end{array}$

- Albrecht von Haller und der praktische Arzt seiner Zeit 253

Marguerite Carozzi, Bonnet, Spallanzani, and Voltaire on Regeneration of Heads in Snails:

a Continuation of the Spontaneous Generation Debate 265

$\begin{array}{lr}\text { Jean Jacques Dreifuss, Moritz Schiff et la vivisection } & 289\end{array}$

Adolf Faller, Zur Diskussion um das Stensen-Experiment 19

Charles Hahn, Fondements historiques du principe de la reconstruction
en chirurgie vasculaire

Huldrych M. Koelbing, Hippokratische Medizin (Cinquième Colloque International Hippocratique), ein internationales Kolloquium in Berlin 161

- Zur Erinnerung an Heinrich Buess (1911-1984) 3

- Remarques à propos de l'évolution des connaissances sur le cerveau 315

Samuel S. Kottek, Physicians and healing personnel in the works of Flavius Josephus $\quad 47$

Andreas-Holger Maehle, Johann Jakob Wepfers experimentelle Toxikologie 7

Philippe Mudry, Médecins et spécialistes $\quad 329$

Jackie Pigeaud, Homo quadratus. Variations sur la beauté et la santé dans la médecine antique

Marie-Louise Portmann, Der Basler Arzt Theodor Zwinger III (1658-1724) und seine Arbeit über ein langes Leben

Luzius von Rechenberg und Huldrych M. Koelbing, Hufelands Opiumtherapie im zeitgenössischen Vergleich $\quad 97$

Olivier Rieppel, The dream of Charles Bonnet (1720-1793) 359

Jacques Roger, Lamarck et Jean-Jacques Rousseau 369

Gerhard Rudolph, Amor medicabilis herbis. Un essai au XVIII ${ }^{e}$ siècle de se guérir de l'érotomanie par la Ciguë

B. Rüttimann, Souvenirs des guerres napoléoniennes: points de vue des blessés et de leurs médecins

Werner Sackmann, James Israel: Meine Reise zum Sultan (1915). Bemerkungen zum Tagebuch einer ärztlichen Mission

Francis Schiller, The Mystique of the Frontal Lobes

Michael Shepherd, Sozialgeschichte: die Geschichte eines Begriffs

Pieter Smit, The Swiss zoologist Rudolf Burckhardt (1866-1908), pioneer in biohistory

Antoinette Stettler, Geschichtliches über den Schmerz in Pathologie, Nervenphysiologie und Mentalität 
Owsei Temkin, Hippocrates as the Physician of Democritus

Jean Théodoridès, Le personnage du médecin dans l'œuvre romanesque de Stendhal

Charlotte Triebel-Schubert, Das naturphilosophische Konzept bei Johann Christian Friedrich Harless

Dora B. Weiner, Philippe Pinel, linguist: his work as translator and editor

\section{Buchbesprechungen/Book Reviews}

Otto Baur, Otto Glandien (Ed.), Zusammenhang. Festschrift für Prof.Dr.Dr. Marielene Putscher (Ackerknecht)

Mary A.B. Brazier, A History of Neurophysiology (Stettler) 181

Blas Bruni Celli, Bibliografía Hipocrática (Bloch) 513

Judith Bryce, Cosimo Bartoli. The Career of a Florentine Polymath (Stettler) 535

Willem F.Daems, Johann Anton Grass (Haffter) 518

Zum Werk Leonhard Eulers (Neuenschwander) 534

Paul Feyerabend/Christian Thomas (Ed.), Kunst und Wissenschaft (Stettler) 523

Arndt Fleischer, Patentgesetzgebung und die chemisch-pharmazeutische Industrie im deutschen Kaiserreich (Schramm)

W. Gerlach, Otto Hahn. Ein Forscherleben (Schramm)

Danielle Gourevitch, Le Mal d'être femme. La femme et la médecine dans la Rome antique (Haffter)

Marie-Louise Portmann, Die Heilmittel der Hildegard von Bingen (Daems) 189

Wolfgang Hömberg, Der norddeutsche Bronzemörser im Zeitalter von Gotik und Renaissance (Ledermann)

Rio Howard, La bibliothèque et le laboratoire de Guy de la Brosse au Jardin des Plantes à Paris (Mayer)

Arthur E. Imhof (Ed.), Der Mensch und sein Körper von der Antike bis heute (Haffter)

Andor Kertész, Georg Cantor, Schöpfer der Mengenlehre (Neuenschwander)

Huldrych M. Koelbing, Christian Sigismund Fingers Dissertation «Über den schädlichen Einfluß von Furcht und Schreck bei der Pest» (Stettler)

Johannes Krähe, Die Diskussion um den ärztlichen Kunstfehler (Stettler)

Jochen Kreidel, Johann Heinrich Dierbach (Sehramm)

Fridolf Kudlien, Ärzte im Nationalsozialismus (Haffter)

Franz-Josef Kuhlen, Zur Geschichte der Schmerz-, Schlaf- und Betäubungsmittel in Mittelalter und früher Neuzeit (Sigron)

Liselotte Kuntner, Die Gebärhaltung der Frau (Ackerknecht)

François Ledermann, Bibliographie des ouvrages suisses de pharmacopée (Daems)

Leopoldina-Meeting, Biologische Grundlagen der Geschichtlichkeit des Menschen (Haffter)

J. M. Lopez Pinero, M. Seoane y la introduccion en Espania del sistema sanitario liberal (Ackerknecht)

William H. MacNeill, Plagues and Peoples (Stettler) 
F. K. Mathis, Ist die schwarze Köchin da? (Haffter) 186

C. A. Meier, Der Traum als Medizin (Haffter) 517

Christoph Meinel, In physicis futurum saeculum respicio (Druey) 529

Michler/Benedum, Einführung in die Medizinische Fachsprache (Balmer) 518

Konrad Moll, Der junge Leibniz (v. Meyenn) 530

Maria Teresa Monti, Catologo del Fondo Haller della Biblioteca Nazionale Braidense di Milano (Boschung) 514

Axel Heinrich Murken, Lehrbuch der medizinischen Terminologie (Haffter) 187

Alireza Djafari Naini, Geschichte der Zahlentheorie im Orient, im Mittelalter und zu Beginn der Neuzeit (Neuenschwander) 533

Johannes Oehme, Pädiatrie im 18. Jahrhundert (Portmann) 180

Walter Pagel, The Smiling Spleen, Paracelsianism in Storm and Stress (Daems) 188

Ortrun Riha, Meister Alexanders Monatsregeln (Fehlmann) 519

Roche Lexikon Medizin (Haffter) 188

Albert von Rohr, Die Medizinische Poliklink der Universität Zürich 1835 bis 1983

(Ackerknecht) 511

George Rosen, The Structure of American Medical Practice 1875-1941 (Ackerknecht) 175

Heinrich Schipperges, Historische Konzepte einer theoretischen Pathologie (Stettler) 523

Werner H.Stoecklin, Toktok. Am Rand der Steinzeit auf Neuguinea (Ackerknecht) 178

Alfred Stückelberger, Vestigia Democritea (Druey) 527

Friedrich Tiedemann, Das Hirn des Negers mit dem des Europäers und $\begin{array}{ll}\text { Orang-Outangs verglichen (Boschung) } & 515\end{array}$

Ulrich Tröhler, Der Nobelpreisträger Theodor Kocher (Ackerknecht) 177

Helmut Vogt, Der Arzt am Krankenbett (Haffter) 186

Dederick C. Ward and Albert V. Carozzi, Geology Emerging (Rieppel) 532

Herbert Will, Die Geburt der Psychosomatik. Georg Groddek, der Mensch und
Wissenschaftler (Haffter)

$\begin{array}{ll}\text { Mitteilungen } & 537\end{array}$ 